\title{
Ovarian stimulated cycle: not a better alternative for women without ovulation disorder in intrauterine insemination
}

\author{
Kemei Zhang ${ }^{1}$, Yinjiao Shi ${ }^{1}$, Ensheng Wang ${ }^{1}$, Li Wang ${ }^{1}$, Qingbo Hu ${ }^{1}$, Yibo Dai ${ }^{1}$, \\ Haiyan $X u^{1}$, Jiaou Zhang ${ }^{1}$, Ping Jin ${ }^{1}$, Xueqin Chen ${ }^{1}$ and Jing Shu ${ }^{1}$ \\ ${ }^{1}$ Reproductive Medicine Center, Ningbo First Hospital, Zhejiang 315010, China \\ Correspondence to: Jing Shu, email: 13362482006@163.com
}

Keywords: clinical pregnancy rate, intrauterine insemination (IUI), ovarian stimulated cycle, natural cycle, non-ovulation disorder

Received: June 22, 2017

Accepted: August 27, 2017

Published: October 23, 2017

Copyright: Zhang et al. This is an open-access article distributed under the terms of the Creative Commons Attribution License 3.0 (CC BY 3.0), which permits unrestricted use, distribution, and reproduction in any medium, provided the original author and source are credited.

\section{ABSTRACT}

To explore the related factors on the clinical pregnancy outcome in intrauterine insemination, a retrospective study was conducted on the clinical data of $\mathbf{5 8 0}$ cycles for 301 infertile couples who were treated with intrauterine insemination. The female age, male age, duration of infertility, treatment protocols, endometrial thickness and sperm parameters were compared between pregnant group and non-pregnant group. The results showed that there were statistical differences in female age, duration of infertility and endometrial thickness between the two groups. The pregnancy rate was $19.34 \%$ in Group A (female age $\leq 30$ y) compared with $10.91 \%$ in Group B (female age $>30 \mathrm{y}$ ). The pregnancy rate was $18.44 \%$ when the duration of infertility $\leq 2$ years, which was higher than another group $10.73 \%$ when the duration of infertility $>2$ years. Group analysis according to endometrial thickness (Group1: < 8 mm; Group 2: $\geq 8 \mathrm{~mm}$ and $\leq 12 \mathrm{~mm}$; Group 3: $>12 \mathrm{~mm}$ ) demonstrated significant differences in clinical pregnancy rate $(7.41 \%, 18.00 \%$ and $11.48 \%$ respectively $)$. For those infertile female without ovulation failure, the higher clinical pregnancy rates were observed in patients undergoing intrauterine insemination in natural cycle $16.12 \%$ when compared with the patients in ovarian stimulated cycles $10.48 \%$. Thus, we demonstrate that the pregnancy rate is related with female age, duration of infertility and endometrial thickness. The ovarian stimulated cycle couldn't improve the pregnancy outcome for those women without ovulation disorder in intrauterine insemination.

\section{INTRODUCTION}

Intrauterine insemination (IUI) is the first-line approach for infertile couples in the assisted reproductive treatment (ART) procedures, which is used widely for a broad range of indications in the reproductive medicine. It is commonly applied to the infertile couples diagnosed with the mild male factor, endometriosis, ovulation failure and unexplained factors [1]. Treatment with IUI is simple, less invasive and less expensive, with a lower multiple delivery rate and lower complication than in vitro fertilization (IVF)/ intracytoplasmic sperm injection (ICSI) [2, 3]. Therefore, IUI technology is widely used around the world. The data from the European Society of Human Reproduction and Embryology
(ESHRE) showed that 162,843 IUI cycles were performed in 2009 compared with 135,621 IVF cycles during the same period [2]. However, the pregnancy rate per cycle with IUI is otherwise compared with IVF/ICSI [2, 4]. The sperm preparation techniques have been improved much in recent decades [5, 6], however, the clinical pregnancy rate with IUI remains unchanged [7, 8]. According to the reports from a large number of reproductive centers, the clinical pregnancy rate with IUI per cycle is between $11.4 \%$ and $12.6 \%$ [1]. Thus, how to improve the outcomes of treatment with IUI still remains elusive for all reproductive doctors. Here, data from 580 cycles for 301 infertile couples in our center was analyzed to define which the following factors, including female age, male age, duration of infertility, treatment protocol, endometrial 
thickness, sperm parameters and treatment cycles, contribute to the positive clinical pregnancy outcomes.

\section{RESULTS}

A total of 580 IUI cycles from 301 couples were enrolled in the study from January 1, 2015 to February 28, 2017, including 392 cycles in primary infertility and 188 cycles in secondary infertility. According to the statistics, 121 couples performed one IUI cycle, and 109 couples as well as 40 couples performed two and three cycles. Only 31 couples performed more than four IUI cycles. The mean age of females was 30.3 years old (varied from 20 to 45), and it was 31.6 years old in males (varied from 23 to 46 ). The mean infertility duration was 2.57 years (varied from 1 to 10). There were 89 cases diagnosed as clinical pregnancy. The clinical pregnancy rate was $15.34 \%(89 / 580)$, the abortion rate was $16.85 \%(15 / 89)$, the twin pregnancy rate was $6.52 \%(3 / 89)$, and the ectopic pregnancy rate was $4.49 \%(4 / 89)$.

As presented in Table 1, the infertile females were significantly younger in pregnant group $(29.36 \pm 3.16)$ than those in non-pregnant group $(30.45 \pm 3.68)$, the difference of which was statistical $(p<0.01)$. There was no difference in the age of males between pregnant group (31.24 \pm 4.09$)$ and non-pregnant group $(31.67 \pm 4.10)$ $(p>0.05)$. The clinical pregnancy rate was $19.34 \%$ $(59 / 305)$ in females whose ages were below 30 years old, and that was $10.91 \%(30 / 275)$ in those females who were above $30(p<0.01)$, as shown in Table 2 . The clinical pregnancy rate had no significant difference between the males whose ages were below 30 years old and those whose ages were above $30(18.03 \%$ to $13.39 \%, p>0.05)$.

The duration of infertility was significantly different between the pregnant group and the non-pregnant group $(2.12 \pm 1.32$ years to $2.65 \pm 1.60$ years, $p<0.01)$, as presented in Table 1 . The clinical pregnancy rate was $18.44 \%(64 / 347)$ in the group whose infertility duration was less than 2 years, and was $10.73 \%(25 / 233)$ in those whose duration were more than 2 years $(p<0.05)$, as presented in Table 2.

Our results showed that women with normal menstruation were performed 452 cycles, including 242 natural cycles and 210 ovarian stimulated cycles. The clinical pregnancy rate was higher in natural cycles than in ovarian stimulated cycles (16.12\% to $10.48 \%)$. However, the difference was not statistical $(p>0.05)$. As shown in Table 3, other women with ovulation dysfunction were performed 210 ovarian stimulated cycles. The clinical pregnancy rate was $13.33 \%$ in $\mathrm{CC} / \mathrm{CC}+\mathrm{Gn}$ group, $23.26 \%$ in letrozole/ letrozole $+\mathrm{Gn}$ group, and $33.33 \%$ in Gn group $(p>0.05)$.

The endometrial thickness on hCG day was $9.94 \pm 1.86 \mathrm{~mm}$ in pregnant group and $9.41 \pm 2.17 \mathrm{~mm}$ in non-pregnant group $(p<0.05)$. As shown in Table 4, the cases were divided into three groups according to the membrane on the day of hCG trigger (Group1: $<8 \mathrm{~mm}$; Group 2: 8-12 mm; Group 3:> $12 \mathrm{~mm}$ ). The pregnancy rates were statistically different among the three groups $(7.41 \%, 18.00 \%, 11.48 \%, p<0.05)$.

The progressive motility of males' sperm was $31.72 \pm 9.27 \%$ in the pregnant group and $32.32 \pm 9.68 \%$ in the non-pregnant group $(p>0.05)$. As presented in Table 5 , the cases were divided into three groups according to the progressive motility of males' sperm (Group 1: $\leq 20 \%$; Group 2: $20-32 \%$; Group3: $\geq 32 \%$ ), and the pregnancy rates were not significantly different $(11.94 \%$ 、 $16.39 \% 、 15.27 \%, p>0.05)$.

As presented in Table 6, the cases were divided into four groups according to the times of IUI cycles (first cycle, second cycle, third cycle and $\geq$ forth cycle), and the pregnancy rates were not significantly different $(14.09 \%, 14.44 \%, 23.94 \%, 12.90 \%, p>0.05)$. And the accumulated pregnancy rates were $14.09 \%, 30.63 \%$ 、 $61.15 \%, 76.72 \%$, respectively. Among the pregnant group (89 women got pregnant totally), 47.19\% (42/89) patients got pregnant in the first time, and $29.21 \%(26 / 89)$ patients as well as $19.10 \%(17 / 89)$ patients got pregnant in the second and third time, respectively. Only $4.49 \%$ (4/89) patients got pregnant four times later, as shown in Figure 1.

\section{DISCUSSION}

The number of infertile couples has increased a lot in recent years due to the environmental pollution and work pressure. Thanks to the rapid development of ART, more and more infertile couples choose this technique to resolve their reproductive problems. IUI is the first-line treatment during ART procedure [9], which makes sperm bypass the cervical-barrier to increase the number of sperm surrounding the egg. The sperm are washed to remove the inactive sperm, immature germ cells, microorganisms, white cells and antigen proteins. By this way, the activity and quality of sperm are meliorated enormously. IUI is a simpler, safer and cheaper treatment compared with IVF/ ICSI. However, the success rate of IUI is lower than other ART, which limits the application of this technology. In this study, we find out some factors which contribute to the outcome of IUI treatment.

Age

In the present research, we found that the female age was different between pregnant group and nonpregnant group. The women were younger in pregnant group than those in non-pregnant group, in agreement with the literatures [7, 10-13]. The differences were statistically significant. When the woman was older, the clinical pregnancy rate with IUI was lower accordingly. Otherwise, there was no difference in the effect of male age on the pregnancy rate. As we know, female age is 
Table 1: Comparison of the female age, male age, duration of infertility, endometrial thickness on hCG day and PR between pregnant group and non-pregnant group

\begin{tabular}{lccccc}
\hline & Female age (y) & Male age (y) & $\begin{array}{c}\text { Duration of } \\
\text { infertility (y) }\end{array}$ & $\begin{array}{c}\text { Endometrial } \\
\text { thickness (mm) }\end{array}$ & PR (\%) \\
\hline Pregnant group & $29.36 \pm 3.16$ & $31.24 \pm 4.09$ & $2.12 \pm 1.32$ & $9.94 \pm 1.86$ & $31.72 \pm 9.27$ \\
Non-pregnant group & $30.45 \pm 3.68$ & $31.67 \pm 4.10$ & $2.65 \pm 1.60$ & $9.41 \pm 2.17$ & $32.32 \pm 9.68$ \\
$P$ value & $0.009^{* *}$ & 0.362 & $0.003^{* *}$ & $0.032^{*}$ & 0.592 \\
\hline
\end{tabular}

Table 2: The clinical pregnancy rate according to female age, male age and duration of infertility

\begin{tabular}{|c|c|c|c|c|c|c|}
\hline & \multicolumn{2}{|c|}{ Female age $(y)$} & \multicolumn{2}{|c|}{ Male age $(y)$} & \multicolumn{2}{|c|}{ Duration of infertility $(y)$} \\
\hline & $\leq \mathbf{3 0}$ & $>\mathbf{3 0}$ & $\leq \mathbf{3 0}$ & $>\mathbf{3 0}$ & $\leq 2$ & $>2$ \\
\hline Pregnant group & 59 & 30 & 44 & 45 & 64 & 25 \\
\hline Non-pregnant group & 246 & 245 & 200 & 291 & 283 & 208 \\
\hline Clinical pregnancy rate(\%) & $19.34 \%$ & $10.91 \%$ & $18.03 \%$ & $13.39 \%$ & $18.44 \%$ & $10.73 \%$ \\
\hline$\chi^{2}$ value & \multicolumn{2}{|c|}{7.921} & \multicolumn{2}{|r|}{2.343} & \multicolumn{2}{|c|}{6.386} \\
\hline$P$ value & \multicolumn{2}{|c|}{$0.005^{* *}$} & \multicolumn{2}{|r|}{0.126} & \multicolumn{2}{|c|}{$0.012^{*}$} \\
\hline
\end{tabular}

Table 3: The clinical pregnancy rate according to the treatment protocols

\begin{tabular}{|c|c|c|c|c|c|c|}
\hline \multirow{3}{*}{$\begin{array}{l}\text { Non-ovulation } \\
\text { disorder }\end{array}$} & \multirow{4}{*}{$\begin{array}{l}\text { Pregnant group } \\
\text { Non-pregnant group } \\
\text { Clinical pregnancy } \\
\text { rate }(\%)\end{array}$} & \multirow{2}{*}{$\frac{\text { Natural cycles }}{39}$} & \multicolumn{2}{|c|}{ Ovrian stimulated cycles } & \multirow[t]{2}{*}{$\chi^{2}$ value } & \multirow[t]{2}{*}{$P$ value } \\
\hline & & & \multicolumn{2}{|c|}{22} & & \\
\hline & & 203 & \multicolumn{2}{|c|}{188} & \multirow{2}{*}{3.063} & \multirow{2}{*}{0.080} \\
\hline & & $16.12 \%$ & \multicolumn{2}{|c|}{$10.48 \%$} & & \\
\hline \multirow[t]{4}{*}{$\begin{array}{l}\text { Ovulation } \\
\text { disorder }\end{array}$} & & $\mathrm{CC} / \mathrm{CC}+\mathrm{Gn}$ & $\begin{array}{l}\text { Latrozole/ } \\
\text { latrozole+Gn }\end{array}$ & Gn & \multirow{4}{*}{2.299} & \multirow{4}{*}{0.317} \\
\hline & Pregnant group & 4 & 20 & 4 & & \\
\hline & Non-pregnant group & 26 & 66 & 8 & & \\
\hline & $\begin{array}{l}\text { Clinical pregnancy } \\
\text { rate }(\%)\end{array}$ & $13.33 \%$ & $23.26 \%$ & $33.33 \%$ & & \\
\hline
\end{tabular}

Table 4: The clinical pregnancy rate according to the endometrial thickness on hCG day

\begin{tabular}{lccccc}
\hline & $<\mathbf{8} \mathbf{~ m m}$ & $\mathbf{8 - 1 2} \mathbf{~ m m}$ & $>\mathbf{1 2} \mathbf{~ m m}$ & $\chi^{\mathbf{2}}$ value & $\boldsymbol{P}$ value \\
\hline Pregnant group & 8 & 74 & 7 & & \\
Non-pregnant group & 100 & 337 & 54 & 8.180 & $0.017^{*}$ \\
Clinical pregnancy rate (\%) & $7.41 \%$ & $18.00 \%$ & $11.48 \%$ & & \\
\hline
\end{tabular}

an independent factor affecting pregnancy outcome $[7,14]$. When the women become older, especially over 35 years old, the amount of oocyte exhausts rapidly. The accumulation of metabolites in the body changes the ovarian environment, such as the mutations in DNA and shortening of telomeres, which physiologically declines the quality of oocytes, increasing the chance of infertility [15-20]. It has been reported that the optimal reproductive age for women is 20 to 30 years old, and the ability of reproduction declines rapidly after 30 years old $[7,14]$. In modern society, especially in some developed cities, many couples delay the timing of having children, which brings a lot of reproductive problems. The spontaneous abortion rate and fetal malformation rate go up accompanied by the maternal age. It has been shown that the pregnancy rate prominently reduces when the age of women is over 37 years old and is very little when over 40 years old. In our report, there was no effect in the male age on 
Table 5: The clinical pregnancy rate according to the PR before washing

\begin{tabular}{lccccc}
\hline & $\mathbf{2 0} \%$ & $\mathbf{2 0}-\mathbf{3 2} \%$ & $\mathbf{2 3 2} \%$ & $\boldsymbol{\chi}^{\mathbf{2}}$ value & $\boldsymbol{P}$ value \\
\hline Pregnant group & 8 & 39 & 42 & & \\
Non-pregnant group & 59 & 199 & 233 & 0.798 & 0.671 \\
Clinical pregnancy rate (\%) & $11.94 \%$ & $16.39 \%$ & $15.27 \%$ & & \\
\hline
\end{tabular}

Table 6: The clinical pregnancy rate according to the operation times

\begin{tabular}{|c|c|c|c|c|c|c|}
\hline & First cycle & Second cycle & Third cycle & $\geq$ Fourth cycle & $\chi^{2}$ value & $P$ value \\
\hline Pregnant group & 42 & 26 & 17 & 4 & & \\
\hline Non-pregnant group & 256 & 154 & 54 & 27 & 4.655 & 0.199 \\
\hline Clinical pregnancy rate $(\%)$ & $14.09 \%$ & $14.44 \%$ & $23.94 \%$ & $12.9 \%$ & & \\
\hline & \multicolumn{2}{|c|}{ One cycle } & Two cycles & Three cycles & \multicolumn{2}{|c|}{$\geq$ four cycles } \\
\hline Pregnant couples & \multicolumn{2}{|c|}{42} & 68 & 85 & \multicolumn{2}{|c|}{89} \\
\hline Infertile couples & \multicolumn{2}{|c|}{298} & 222 & 139 & \multicolumn{2}{|c|}{116} \\
\hline Accumulative pregnancy rate & \multicolumn{2}{|c|}{$14.09 \%$} & $30.63 \%$ & $61.15 \%$ & \multicolumn{2}{|c|}{$76.72 \%$} \\
\hline
\end{tabular}

the pregnancy rate. Therefore, we should consider the female age as an important factor while determining the therapeutic regimen.

\section{Duration of infertility}

In our study, we found that the duration of infertility was different between pregnant group and non-pregnant group. It was significantly shorter in pregnant group than in non-pregnant group. Furthermore, the clinical pregnancy rate per cycle of couples was higher when their duration of infertility was below 2 years, compared with couples over 2 years. This report is consistent with previously reported [11]. Thus we come to conclusion that the duration of infertility is highly associated with the pregnancy rate. The pregnancy rate per cycle with IUI would decrease when the duration of infertility is longer.

\section{The endometrial thickness on hCG day}

According to the statistics, the average endometrial thickness was different between pregnant group and non-

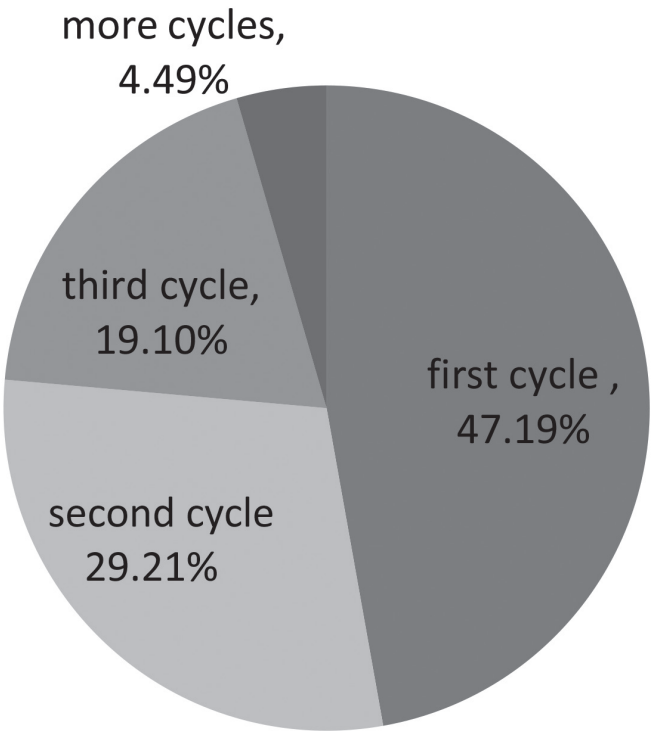

Figure 1: The clinical pregnancy rate according to the cycles in pregnant patients. 
pregnant group. When the patients were divided into three groups according to the membrane on the day of hCG trigger (Group1: < $8 \mathrm{~mm}$; Group 2: 8-12 mm; Group 3: $>12 \mathrm{~mm}$ ), we found that the pregnancy rate was highest in the group when the endometrial thickness ranged from 8 to $12 \mathrm{~mm}$ compared with other two groups, which was consistent with the previous studies $[21,22]$. Weissman et al. [23] reported that the pregnancy rate is proportional to the endometrial thickness. However, the pregnancy rate and planted rate decline when the endometrial thickness is over $14 \mathrm{~mm}$. It will lead to implantation failure when the endometrial thickness is too thick or too thin $[23,24]$. Thin endometrium was mostly caused by the low estrogen, endometrial damage or inflammation. Low estrogen usually dues to the poor oocyte quality and poor ovarian function, which may decline the pregnancy rate by affecting the quality of embryo and endometrial receptivity. On the other hand, the implantation is affected by the endometrial damage or inflammation in another way. The decline of the pregnancy rate might be related to the endometrial damage caused by the transfer tube with IUI when the endometrial thickness is too thick.

\section{Treatment protocol: natural cycle and ovarian stimulated cycle}

In current study, we found that there was no statically difference in the clinical pregnancy rate per cycle with IUI between natural cycle and ovarian stimulated cycle for those women with regular menstruations and normal ovulation. It has been revealed that ovarian stimulated treatment could not improve the outcome of the IUI for the infertile women with non-ovulation disorder. At the same time, there was no difference in the pregnancy rate among groups with different ovarian stimulated protocols. It was announced that the pregnancy rate with ovarian stimulated cycles is higher than that with natural cycles $[25,26]$. In our opinion, the opposite conclusion is due to the different cohort chosen in the different research. In the previous study, people with or without ovulation dysfunction were combined for the statistics. The ovarian stimulation treatment would be efficient obviously for those women with the ovulation dysfunction, whom the natural cycle could not be used for. Therefore, it is not difficult to come to a false conclusion that ovarian stimulated cycle is better than natural cycle.

As shown in Table 3, the clinical pregnancy rate in natural cycle group is higher than in ovarian stimulated cycle group $(16.12 \%$ to $10.48 \%)$, although without statistics difference (Table 3). In the clinic, we found that the infertile couples with mild male factors, such as sexual dysfunction, preferred to choose natural cycle in IUI treatment. Those infertile patients were easy to get pregnant in IUI cycle. In our opinion, that's the reason why the outcome in natural cycle group was better than in ovarian stimulated cycle group.
In general, the ovarian stimulated cycle is not a better alternative compared with the natural cycle for the women without ovulation disorder in IUI.

\section{Sperm parameter}

In our research, there was no difference in PR before sperm was washed between the pregnant group and nonpregnant group. The pregnancy rate was highest in the group when the PR was from $20 \%$ to $32 \%$. Stone et al. [14] also reported that sperm motility in inseminate was a major determinant of outcome, with $\mathrm{PR}<20 \%$ significantly decreasing the pregnancy outcome. As we know, the IUI treatment is a technology through putting sperm into the uterine to increase the amount of sperm around the egg. Thus, male patients with mild asthenospermia are the best subjects who receive this treatment. However, when the activity of sperm declines further, the amount of sperm can hardly reach the enough concentration to fertilize the egg. On the other hand, the female can get pregnant without IUI if the sperm are normal. In this point, there must be some other factors which affect the pregnancy outcome for those infertile couples, such as sexual dysfunction, fallopian tube inflammation, endometriosis and unexplained reasons. It is very difficult to get pregnant with IUI for people with those reasons mentioned above, except sexual dysfunction. That is why the pregnancy rate was lower with IUI in the group when the sperm was normal compared with the group when the sperm was abnormal mildly. We can see the tendency even if there was no significant difference among three groups. We will expand the sample size to confirm this regularity in our future investigation.

\section{Treatment cycles}

Most of the infertile couples performed 1-3 cycles with IUI treatment in our reproductive center. Seven couples performed 5cycles, and two couples performed 6 cycles. It is suggested that the infertile couples with unexplained reasons should be treated with IUI for at least 3 times before turning to further assisted reproductive technique, such as IVF/ICSI.

Our data showed that the cumulative pregnancy rate increased gradually with the increase of cycles. The cumulative pregnancy rate was up to $61.15 \%$ when the couples preformed 3 cycles with IUI. According to the analysis in the pregnant group, $47.19 \%$ patients got pregnant in the first time, and $29.21 \%$ patients as well as $19.10 \%$ patients got pregnant in the second and third time, respectively. Only $4.49 \%$ patients got pregnant four times later. Although the cumulative pregnancy rate increases gradually by the increase of cycles, the chances of getting pregnant per cycle diminishes conversely, which is similar with Dinelli et al. [7]. We thought that the pregnancy rate was related to the causes of infertile couples. Some 
patients with mild female factor, cervical factor and mild endometriosis can get pregnant with IUI in the first three times. There are still some potential factors which are hardly to be discovered by the current technology. It is difficult to get pregnant with IUI by increasing the cycles for these patients.

Collectively, the IUI treatment is a kind of simple, cheap and invasive technique compared with IVF/ICSI. There is lower success rate but less intervention in the reproductive process with IUI compared with other assisted reproductive techniques. We suggest those infertile couples who may get pregnant with IUI to perform this first-line technology in reproductive treatments. More importantly, natural cycle may get a better outcome for those people with non-ovulation dysfunction in the IUI treatment. In other words, the ovarian stimulated cycle is not a better alternative for infertile women with normal ovulation.

\section{MATERIALS AND METHODS}

\section{Study design}

A retrospective study was performed by reviewing the clinical data of 580 IUI cycles from 301 couples at the Reproductive Center of Ningbo First Hospital in China during January 2015 and February 2017. The inclusion criteria for IUI included: infertility for at least one year, at least one patent fallopian tube. Duration of infertile: from the time since a couple have sex without any contraception. Informed consent was obtained from all subjects. The study protocol was approved by the Institutional Review Board of Ningbo First Hospital, China. All methods were performed in accordance with the approved guidelines.

\section{Treatment protocols}

\section{Natural cycle}

Natural cycle was administered in females whose menstrual cycles were regular. Intrauterine insemination was administered according to the peak of the luteinizing hormone (LH) which was measured day by day since the diameter of follicle got to $16-18 \mathrm{~mm}$.

Ovulation induction was performed as the following protocol:

Clomiphene citrate ( CC) 50-100 mg/day starting from day 3-5 for 5 days.

Letrozole (LE) 2.5-5.0 mg/day from day 3-5 for 5 days.

HMG 37.5-75 IU/day starting from day 3-5 for a variable duration depending on the response.

CC combined with HMG - CC 50-100 mg/day starting from days 3-5 for 5 days followed by the addition of 37.5-75 IU of HMG for a variable duration depending on the response.

LE combined with HMG - LE 2.5-5.0 mg/day starting from day $3-5$ for 5 days followed by the addition of 37.5-75 IU of HMG for a variable duration depending on the response.

Intrauterine insemination (IUI) was administered according to the peak of the luteinizing hormone. And the cycle would be canceled to avoid the ovarian hyperstimulation syndrome (OHSS) if the estradiol (E2) was higher than $1500 \mathrm{pg} / \mathrm{ml}$ or the mature follicles were more than 3 .

\section{Operative time}

When at least one mature follicle had a diameter of $18 \mathrm{~mm}$ or more and the endometrial thickness achieved $7 \mathrm{~mm}$, we triggered ovulation with intramuscular injection of urinary human chorionic gonadotrophin (hCG) (5000-10 000 IU), or hypodermic injection of recombinant human chorionic gonadotropin alfa (Ovidrel, $0.25 \mathrm{mg}$ ), or hypodermic injection of Triptorelin $(0.1 \mathrm{mg})$. Insemination was performed 36-40 hours after injection.

\section{Semen treatment}

Semen was collected by masturbation after abstinence for 3-7 days and prepared with two-layer density gradient centrifugation after liquefaction. Mechanical method was used for abnormal semen liquefaction. The volume of washed semen sample used for insemination was $0.3-0.5 \mathrm{ml}$.

\section{Luteal phase support}

The luteal phase was used routinely in all patients, starting from the day since IUI was performed. It consisted of Duphaston (Dydrogesterone Tablets, $20 \mathrm{mg}$ /day, Abbott, Netherlands) for 14 days. A blood test for hCG assay was performed in 14 days after insemination to confirm whether pregnancy had occurred. In women with positive hCG, ultrasound examination was performed at 7 weeks' gestation to confirm fetal viability. A clinical pregnancy was defined as one in which there was ultrasonographic evidence.

\section{Statistical analysis}

The data expresses the means \pm SD. The baseline differences between the two groups were analyzed by Student's $t$ test. Pearson's Chi-square test was used to compare the ratios between groups. A value of $p$ less than 0.05 was considered statistically significant. The data was analyzed using the Statistical Package for the Social Sciences (SPSS) for Windows (version 19.0). 


\section{Author contributions}

K.Z. and J.S. designed the research; Y.S., E.W., L.W., Q.H. and Y.D. collected the cases; K.Z., H.X., J.Z., P.J., X.C. and J.S. analyzed the data; K.Z. wrote the paper.

\section{ACKNOWLEDGMENTS}

We thank Dr. Lina Zhang and Yunfei Lu for providing help in Statistics. We also thank Rozita Petri for providing help in grammar.

\section{CONFLICTS OF INTEREST}

The authors declare no conflicts of interest to disclose.

\section{FUNDING}

This work was supported by the Natural Science Foundation of Ningbo (2016A610161) and the Natural Science Foundation of Ningbo(2016A610170).

\section{REFERENCES}

1. ESHRE Capri Workshop Group. Intrauterine insemination. Hum Reprod Update. 2009; 15:265-277.

2. Ferraretti AP, Goossens V, Kupka M, Bhattacharya S, de Mouzon J, Castilla JA, Erb K, Korsak V, Nyboe Andersen A. Assisted reproductive technology in Europe, 2009: results generated from European registers by ESHRE. Hum Reprod. 2013; 28:2318-2331.

3. Abdelkader AM, Yeh J. The potential use of intrauterine insemination as a basic option for infertility: a review for technology-limited medical settings. Obstet Gynecol Int. 2009; 2009:584837.

4. Fauser BC, Nargund G, Andersen AN, Norman R, Tarlatzis B, Boivin J, Ledger W. Mild ovarian stimulation for IVF: 10 years later. Hum Reprod. 2010; 25:2678-2684.

5. Urry RL, Middleton RG, Jones K, Poulson M, Worley R, Keye W. Artificial insemination: a comparison of pregnancy rates with intrauterine versus cervical insemination and washed sperm versus serum swim-up sperm preparations. Fertil Steril. 1988; 49:1036-1038.

6. Boomsma CM, Heineman MJ, Cohlen BJ, Farquhar C. Semen preparation techniques for intrauterine insemination. Cochrane Database Syst Rev. 2007; CD004507.

7. Dinelli L, Courbiere B, Achard V, Jouve E, Deveze C, Gnisci A, Grillo JM, Paulmyer-Lacroix O. Prognosis factors of pregnancy after intrauterine insemination with the husband's sperm: conclusions of an analysis of 2,019 cycles. Fertil Steril. 2014; 101:994-1000.

8. Atasever M, Kalem MN, Hatirnaz S, Hatirnaz E, Kalem Z, Kalaylioglu Z. Factors affecting clinical pregnancy rates after IUI for the treatment of unexplained infertility and mild male subfertility. J Turk Ger Gynecol Assoc. 2016; 17:134-138.

9. Duran HE, Morshedi M, Kruger T, Oehninger S. Intrauterine insemination: a systematic review on determinants of success. Hum Reprod Update. 2002; 8:373-384.

10. Merviel P, Heraud MH, Grenier N, Lourdel E, Sanguinet $P$, Copin $\mathrm{H}$. Predictive factors for pregnancy after intrauterine insemination (IUI): an analysis of 1038 cycles and a review of the literature. Fertil Steril. 2010; 93:79-88.

11. Tomlinson MJ, Amissah-Arthur JB, Thompson KA, Kasraie JL, Bentick B. Prognostic indicators for intrauterine insemination (IUI): statistical model for IUI success. Hum Reprod. 1996; 11:1892-1896.

12. Steures P, van der Steeg JW, Mol BW, Eijkemans MJ, van der Veen F, Habbema JD, Hompes PG, Bossuyt PM, Verhoeve HR, van Kasteren YM, van Dop PA. Prediction of an ongoing pregnancy after intrauterine insemination. Fertil Steril. 2004; 82:45-51.

13. Kamath MS, Bhave P, Aleyamma T, Nair R, Chandy A, Mangalaraj AM, Muthukumar K, George K. Predictive factors for pregnancy after intrauterine insemination: A prospective study of factors affecting outcome. J Hum Reprod Sci. 2010; 3:129-134.

14. Stone BA, Vargyas JM, Ringler GE, Stein AL, Marrs RP. Determinants of the outcome of intrauterine insemination: analysis of outcomes of 9963 consecutive cycles. Am J Obstet Gynecol. 1999; 180:1522-1534.

15. de Lange T. Ending up with the right partner. Nature. 1998; 392:753-754.

16. Keefe DL, Marquard K, Liu L. The telomere theory of reproductive senescence in women. Curr Opin Obstet Gynecol. 2006; 18:280-285.

17. Wolstenholme J, Angell RR. Maternal age and trisomy - a unifying mechanism of formation. Chromosoma. 2000; 109:435-438.

18. Pellestor F, Anahory T, Hamamah S. Effect of maternal age on the frequency of cytogenetic abnormalities in human oocytes. Cytogenet Genome Res. 2005; 111:206-212.

19. Pellestor F, Andreo B, Anahory T, Hamamah S. The occurrence of aneuploidy in human: lessons from the cytogenetic studies of human oocytes. Eur J Med Genet. 2006; 49:103-116.

20. Pellestor F, Andreo B, Arnal F, Humeau C, Demaille J. Maternal aging and chromosomal abnormalities: new data drawn from in vitro unfertilized human oocytes. Hum Genet. 2003; 112:195-203.

21. De Geyter C, Schmitter M, De Geyter M, Nieschlag E, Holzgreve W, Schneider HP. Prospective evaluation of the ultrasound appearance of the endometrium in a cohort of 1,186 infertile women. Fertil Steril. 2000; 73:106-113.

22. Yuan X, Saravelos SH, Wang Q, Xu Y, Li TC, Zhou C. Endometrial thickness as a predictor of pregnancy outcomes in 10787 fresh IVF-ICSI cycles. Reprod Biomed Online. 2016; 33:197-205. 
23. Weissman A, Gotlieb L, Casper RF. The detrimental effect of increased endometrial thickness on implantation and pregnancy rates and outcome in an in vitro fertilization program. Fertil Steril. 1999; 71:147-149.

24. Zhao J, Zhang Q, Li Y. The effect of endometrial thickness and pattern measured by ultrasonography on pregnancy outcomes during IVF-ET cycles. Reprod Biol Endocrinol. 2012; 10:100.
25. Cohlen BJ, Vandekerckhove P, te Velde ER, Habbema JD. Timed intercourse versus intra-uterine insemination with or without ovarian hyperstimulation for subfertility in men. Cochrane Database Syst Rev. 2000; CD000360.

26. Nulsen JC, Walsh S, Dumez S, Metzger DA. A randomized and longitudinal study of human menopausal gonadotropin with intrauterine insemination in the treatment of infertility. Obstet Gynecol. 1993; 82:780-786. 